\title{
TWO-KINK SOLUTION FOR THE HIROTA-RAMANI EQUATION FOR DEGENERATED PARAMETERS
}

\author{
M. A. Knyazev \\ Belarusian National Technical University, \\ 65, Independence Ave., Minsk, 220013, Belarus \\ e-mail: maknyazev@bntu.by
}

(Received July 16, 2018; in final form October 06, 2018)

\begin{abstract}
The Hirota-Ramani equation is considered. Its two-kink solutions are known and may be constructed, for example, by direct methods if the parameters $k_{i}, i=1,2$ of the solution components are not equal to each other. In this paper, such solution is constructed for $k_{1}=k_{2}$.
\end{abstract}

Key words: two-kink solution, Hirota-Ramani equation, degenerated parameters.

DOI: https://doi.org/10.30970/jps.22.4001

PACS number(s): 02.30.Jr, 03.50.Kk

\section{INTRODUCTION AND FORMULATION OF THE PROBLEM}

The $(1+1)$-dimensional Hirota-Ramani equation is a known nonlinear evaluation equation in the partial derivatives. It is used for the description of various phenomena in physics of fluids, plasma physics, solid state physics, quantum field theory. This equation was derived for the first time in [1]. It has the form

$$
u_{t}-u_{x x t}+a u_{x}\left(1-u_{t}\right)=0,
$$

where $a$ is a constant, $a \neq 0, u_{t}=\frac{\partial u}{\partial t}$ and so on.

The problem of solutions to the Hirota-Ramani equation attracts a lot of attention.In $[1,2]$ it was demonstrated that this equation is completely integrable by the inverse scattering transform method. The soliton solutions to this equation were obtained in [3] by the Expfunction method. The various hyperbolic, trigonometric and rational function solutions were constructed in [4] by the $\left(G^{\prime} / G\right)$-expansion method. A modification of this method realized in [5] allowed to construct new additional solutions. Other new solutions were obtained by the $F$-expansion method and an extended version of the Jacobi elliptic functions [6]. The direct rational exponential scheme was applied for constructing the multi-soliton solutions [7]. Simultaneously with the search of new solutions, attention was paid to the study of the properties of this equation. Its Lie symmetries, conservation laws and differential invariants were considered in $[8,9]$.

The known multi-kink solutions to Eq. (1), including its two-kink solution, may be constructed if the condition $k_{i} \neq k_{j}$ is fulfilled, where $k_{i}$ and $k_{j}$ are the parameters of the components of the solution $[7,10]$. These components are not one-kink components of a multi-kink solution; the parameters mentioned above are used in the construction of a one-kink solution [11].

In this paper, a two-kink solution to the HirotaRamani equation is constructed for the case when $k_{1}=$ $k_{2}$. It is done by the partially modified Hirota method [10] that will be completed by new additional conditions.

Let us introduce a new dependent variable by means of the equation

$$
u(x, t)=\sigma \frac{F_{x}}{F}
$$

where $\sigma$ is a constant determined below, $F=F(x, t)$ is a new unknown function. The arguments of the functions will be dropped out where it is possible. Substitution of Eq. (2) into Eq. (1) results in the following equation:

$$
\begin{aligned}
& F_{x t} F^{3}-F_{x} F_{t} F^{2}-F_{x x x t} F^{3}+F_{t} F_{x x x} F^{2}+3 F_{x t} F_{x x} F^{2}+3 F_{x} F_{x x t} F^{2} \\
& -6 F_{t} F_{x} F_{x x} F-6 F_{x}^{2} F_{x t} F+6 F_{t} F_{x}^{3}+a F_{x x} F^{3}-a F_{x}^{2} F^{2}-a \sigma F_{x x} F_{x t} F^{2} \\
& +a \sigma F_{x} F_{x x} F_{t} F+a \sigma F_{x}^{2} F_{x t} F-a \sigma F_{x}^{3} F_{t}=0 .
\end{aligned}
$$

Now the parameter $\sigma$ may be determined. As we look for a special solution, let us assume that the condition

$$
(6-a \sigma) F_{x}^{3} F_{t}=0
$$

must be fulfilled. It follows from this equation that $\sigma=\frac{6}{a}$. For such $\sigma$ Eq. (3) may be represented in the so called bilinear form

$$
\begin{aligned}
F_{x t} F & -F_{x} F_{t}-F_{x x x t} F+F_{t} F_{x x x}-3 F_{x x} F_{x t} \\
& +3 F_{x} F_{x x t}+a F_{x x} F-a F_{x}^{2}=0 .
\end{aligned}
$$

The next step is typical of direct methods. Let us rep- 
resent $F(x, t)$ as a formal series:

$$
F(x, t)=1+\varepsilon f_{1}+\varepsilon^{2} f_{2}+\varepsilon^{3} f_{3}+\ldots,
$$

where $f_{i}(x, t), i=1,2,3, \ldots$ are new unknown functions and $\varepsilon$ is not a small constant. By substituting Eq. (5) into Eq. (4) and equating the coefficients to zero for each power of $\varepsilon$, we obtain an infinite system of linear partial differential equations for the functions $f_{i}$. To construct a two-kink solution, we need only two functions $f_{1}$ and $f_{2}$ of series (5). To determine them, we should use the first three equations of the infinite system. They have the form:

$$
\begin{aligned}
& f_{1, x t}-f_{1, x x x t}+a f_{1, x x}=0 \\
& f_{2, x t}-f_{2, x x x t}+a f_{2, x x}=f_{1, x} f_{1, t}-f_{1, x x x} f_{1, t}+3 f_{1, x x} f_{1, x t}-3 f_{1, x} f_{1, x x t}+a f_{1, x}^{2}, \\
& f_{3, x t}-f_{3, x x x t}+a f_{3, x x}=f_{1, x} f_{2, t}-f_{1} f_{2, x t}+f_{1, t} f_{2, x}+f_{1} f_{2, x x x t} \\
& -f_{1, x x x} f_{2, t}-f_{1, t} f_{2, x x x}+3 f_{1, x x} f_{2, x t}+3 f_{1, x t} f_{2, x x}-3 f_{1, x} f_{2, x x t}-3 f_{1, x x t} f_{2, x} \\
& -a f_{1} f_{2, x x}+2 a f_{1, x} f_{2, x} .
\end{aligned}
$$

It is clear that for every $i$ the function $f_{i}$ is determined by the previous functions and their derivatives only. Only the first equation of this system is homogeneous, the others are not. If the Hirota method may be applied to solve some equation, the problem of an exact truncation of the series (5) is not essential. But in this paper, we consider a situation when the parameters of the solution are degenerated. That is why we should break the series in a special way and find appropriate conditions for the parameters of the solution.

\section{ONE-KINK SOLUTION}

Let us consider the one-kink solution. This solution is well-known (see, for instance, [7]). We construct it once again to write down a dispersive relation explicitly. Let us represent the function $f_{1}$ in the form

$$
f_{1}(x, t)=\exp \left(k_{1} x-\omega_{1} t+\eta_{1}^{(0)}\right),
$$

where $k_{1}, \omega_{1}$ and $\eta_{1}^{(0)}$ are the parameters of the solution to be determined. $\eta_{1}^{(0)}$ is an initial phase shift and without the loss of generality we may equal it to zero. By substituting Eq. (9) into Eq. (6), we can obtain the following relation between $k_{1}$ and $\omega_{1}$ :

$$
\omega_{1}=\frac{a k_{1}}{1-k_{1}^{2}} .
$$

This is the dispersion relation mentioned above. According to the Hirota method, the second linearly independent equation for $k_{1}$ and $\omega_{1}$ may be obtained by substituting Eq. (9) into the right-hand side of Eq. (7) and equating it to zero. However, in the case of the HirotaRamani equation, this second equation will be of the same form as Eq. (10). Hence one of the parameters $k_{1}$ and $\omega_{1}$ is arbitrary. Let it be $k_{1}$.
Now the one-kink solution may be written as

$$
\begin{aligned}
u(x, t) & =\sigma \frac{f_{1, x}}{1+f_{1}}=\frac{6 k_{1}}{a} \\
& \times\left[1+\tan \left(\frac{k_{1} x-\frac{a k_{1} t}{1-k_{1}^{2}}+\eta_{1}^{(0)}}{2}\right)\right] .
\end{aligned}
$$

\section{TWO-KINK SOLUTION}

Let us construct in the explicit form a two-kink solution for Eq. (1). To do this, we need explicit expressions for the functions $f_{1}$ and $f_{2}$. As for the function $f_{1}$ in the case of a two-kink solution, if the Hirota method or its modifications are used, it is determined in the form

$$
f_{1}(x, t)=\exp \left(\eta_{1}\right)+\exp \left(\eta_{2}\right)
$$

where $\eta_{i}=k_{i} x-\omega_{i} t+\eta_{i}^{(0)}, i=1,2$. For the function $f_{2}$ the coefficients in its various representations contain the combination $k_{1}-k_{2}$. This makes it impossible to construct a two-kink solution when these parameters are degenerated, namely, $k_{1}=k_{2}$. In this paper, the condition $k_{1} \neq k_{2}$ is not required, because no special form for the coefficients in the representation of $f_{2}$ is required.

By substituting Eq. (12) into Eq. (6), we obtain two equations for the parameters $k_{i}$ and $\omega_{i}, i=1,2$. These equations are similar to Eq. (10) and have the form

$$
\omega_{i}=\frac{a k_{i}}{1-k_{i}^{2}}
$$

Let us substitute Eq. (12) into the right-hand side of Eq. (7) and equate to zero the coefficients for the same powers of the exponent. As a result, the following equations can be written

$$
\exp \left(2 \eta_{1}\right): \quad k_{1} \omega_{1}-k_{1}^{3} \omega_{1}+a k_{1}^{2}=0,
$$




$$
\begin{gathered}
\exp \left(2 \eta_{2}\right): \quad k_{2} \omega_{2}-k_{2}^{3} \omega_{2}+a k_{2}^{2}=0, \\
\exp \left(\eta_{1}+\eta_{2}\right): \\
\omega_{2}\left(-k_{1}+k_{1}^{3}-3 k_{1}^{2} k_{2}+3 k_{1} k_{2}^{2}\right)+a k_{1} k_{2} \\
+\omega_{2}\left(-k_{2}+k_{2}^{3}-3 k_{1} k_{2}^{2}+3 k_{1}^{2} k_{2}\right)+a k_{1} k_{2}=0 .
\end{gathered}
$$

Eqs. (14) and (15) are fulfilled due to Eq. (13). Equation (16) is fulfilled on condition that

$$
\omega_{2}\left(-k_{1}+k_{1}^{3}-3 k_{1}^{2} k_{2}+3 k_{1} k_{2}^{2}\right)+a k_{1} k_{2}=0
$$

and

$$
\omega_{1}\left(-k_{2}+k_{2}^{3}-3 k_{1} k_{2}^{2}+3 k_{1}^{2} k_{2}\right)+a k_{1} k_{2}=0 .
$$

These two equations may be considered as additional conditions in the construction of the two-kink solution. They are fulfilled if $\omega_{1}=\omega_{2}=\omega$ and $k_{1}=k_{2}=k$, that is, when the parameters of the two-kink solution are degenerated. This situation is especially interesting to us.

Now, for the function $f_{2}(x, t)$ the following presentation can be applied:

$$
f_{2}=A \exp \left[2\left(k x-\omega t+\eta^{(0)}\right)\right]
$$

Here, for simplicity, $\eta_{1}^{(0)}=\eta_{2}^{(0)}=\eta^{(0)}$ and $A$ is the coefficient to be determined. No special form for $A$ is required (this may be considered as a modification to the Hirota method; for details, see [11]). Under the assumptions taken in this paper, there is only one way to determine $A$. For this purpose, let us substitute Eqs. (12) and (19) into the right-hand side of Eq. (8) and equate it to zero. Unfortunately, direct substitution will be unsuccessful. First of all, we should modify the right-hand side of Eq. (8). To do this, let us apply Eq. (7), then Eq. (8) may be written in the form

$$
\begin{aligned}
f_{3, x t} & -f_{3, x x x t}+a f_{3, x x}=f_{1, x} f_{2, t}+f_{1, t} f_{2, x}-f_{1, x x x} f_{2, t}-f_{1, t} f_{2, x x x} \\
& +3 f_{1, x x} f_{2, x t}+3 f_{1, x t} f_{2, x x}-3 f_{1, x} f_{2, x x t}-3 f_{1, x x t} f_{2, x}+2 a f_{1, x} f_{2, x} \\
& -f_{1} f_{1, x} f_{1, t}+f_{1} f_{1, x x x} f_{1, t}-3 f_{1} f_{1, x x} f_{1, t}+3 f_{1} f_{1, x} f_{1, x x t}-a f_{1} f_{1, x}^{2} .
\end{aligned}
$$

Now let us substitute Eqs. (12) and (19) into the right-hand side of Eq. (20) and equate it to zero. After some algebraic transformations, it will be possible to calculate $A$ :

$$
A=\frac{5 k-2}{k}
$$

Finally, the two-kink solution for the Hirota-Ramani equation for the case when its parameters are degenerated may be written explicitly as

$$
u(x, t)=\sigma \frac{\left(f_{1}+f_{2}\right)_{x}}{1+f_{1}+f_{2}}=\frac{12[k \exp (k x-\omega t)+(5 k-2) \exp 2(k x-\omega t)]}{a\left[1+2 \exp (k x-\omega t)+\frac{5 k-2}{k} \exp 2(k x-\omega t)\right]}
$$

\section{CONCLUSION}

Direct methods for solving the nonlinear equation in the partial derivatives are very powerful. They are simple in the application and clear. That is why they are widely used. But there are certain limits on their application as for any other methods. In this paper, on the example of the Hirota-Ramani equation it is demonstrated how one of such limits may be overcome. The main features of the method have been preserved. We have only added some new conditions and the problem has been solved.
[1] R. Hirota, A. Ramani, Phys. Lett. A 76, 95 (1980).

[2] G. C. Wu, T. C. Xia, Phys. Lett. A 375, 604 (2008).

[3] J. Ji, Appl. Math. Comput. 204, 881 (2008).

[4] Reza Abazari, Rasoul Abazari, Math. Probl. Eng. 2011, 424801 (2011).

[5] E. M. E. Zayed, A. H. Arnous, Int. J. Phys. Sci. 8, 124 (2013).

[6] S. Koonprasert, M. Punpocha, Global J. Pure Applied Mathem. 12, 1903 (2016).

[7] H. O. Roshid, M. N. Alam, Appl. Math. Inf. Sci. 11, 723
(2017).

[8] M. Nadjafikhah, V. Shirvani-Sh, Commun. Nonlin. Sci. Num. Simul. 17, 4064 (2012).

[9] Wei Li, Wenting Li, Fei Wang, Hongqing Zhang, Commun. Nonlin. Sci. Num. Simul. 18, 888 (2013).

[10] M. J. Ablowitz, H. Segur, Solitons and Inverse Scattering Transform, (SIAM, Philadelphia, 1981).

[11] M. A. Knyazev, Kinks in the Scalar Model with Damping, (Technologia, Minsk, 2003) [in Russian]. 


\section{ДВОКІНКОВИЙ РОЗВ'ЯЗОК РІВНЯННЯ ГІРОТИ-РАМАНІ ДЛЯ ВИРОДЖЕНИХ ПАРАМЕТРІВ}

\footnotetext{
М. А. Князев

Білорусъкий начіональний технічний університет, пр. Незалежності, 65, Мінсък, 220013, Білорусъ

Розглянуто рівняння Гіроти-Рамані. Його двокінкові розв'язки можна сконструювати, наприклад, прямими методами, якщо параметри $k_{i}, i=1,2$ компонент розв'язку не дорівнюють один одному. У цій праці отримано розв'язок для випадку $k_{1}=k_{2}$.
} 